\title{
Vegetable fibre: its physical properties
}

\author{
By M. A. Eastwooon, Gastro-intestinal Unit, Western General Hospital, Edinburgh \\ $\mathrm{EH}_{4} 2 \mathrm{XU}$
}

The physical properties exerted by dietary fibre are individual to the age and anatomy of the fruit or vegetable eaten. The physical properties will be determined by the chemistry of the constituent polysaccharides and lignins. In the plant these macromolecules are systematically intermeshed for anatomical and physiological function. This intimate admixture is lost during digestion but the macerated polysaccharides and lignin will retain some of their functional characteristics. These characteristics are important in the function of non-ruminant colon and can be anticipated from the original physiological and anatomical role in the plant.

Following cell division the primary cell wall consists of a watery pectin layer and cellulose. The secondary cell wall, which develops at a much later stage of growth, is formed by fibres running through a structureless matrix. Microfibrils of crystalline or partly crystalline cellulose are distributed with axes at different angles. The interspaces are filled with lignin, hemicelluloses and pectic substances held in a network (Rees, 1967).

\section{Polysaccharides}

Cellulose is essentially a straight chain polymer and in the plant the polymers align side by side, stabilised by hydrogen bonds between hydroxyl groups forming strong intermolecular bonds. This has strength and insolubility and the way in which the chains are packed gives shape to the structure (Liang \& Marchessault, 1957).

Pectin is found in the primary cell walls and in intercellular layers. It is a polyuronic acid polymer, which tends to be branched in growing tissues and less branched when acting in a supporting role. It forms a gel, cross-linked to withstand osmotic repulsions. The association between neighbouring molecules is influenced by the proportion of methyl esters and presence of calcium which neutralises negative charges and influences hydrogen bonds (Gould, Rees, Richardson \& Steele, 1965).

Pectic substances are hydrophilic and adhesive. When a fruit ripens the pectin changes from the insoluble material of the mature plant to a much more watersoluble substance (Worth, I967).

Hemicelluloses are a mixture of linear and highly-branched polysaccharides containing various sugar residues, e.g. xylose, arabinose, glucose and mannose, and $4 \%$ of the residues are uronic acid. Hemicelluloses act as plasticizers. They are intertwined with lignin and laid down around cellulose fibres with a physical admixture through covalent bonds (Aspinall \& Ferrier, 1957). 
Mucilages are polymers containing mainly uronic acid. They have a high capacity for holding water and therefore form reservoirs for water retention in the plant.

Lignin is laid down with cellulose and hemicelluloses producing an essentiallyinert structural material acting as a cementing and anchoring agent. It stiffens the fibre to provide a strong support structure and constitutes approximately $12 \%$ of the the structural material of annual plants. It differs from polysaccharides in composition as it is a phenylpropane polymer; the chemistry of lignins in annual plants is less well documented than that in trees. There is believed to be bonding between the lignins and the neighbouring hemicelluloses.

\section{Physical characteristics of the constituents of plant fibre}

Water adsorption. When water is added to certain polysaccharides they swell to form a semi-rigid, jelly-like mass which holds all the liquid present, i.e. a gel or even a true solution. First water is tightly-bound through hydrogen bonds and this structure then becomes surrounded by loosely-bound water held by dipole attraction and adhesion. As the polymer swells, the spaces in the macromolecule are filled by immobilised water or interstitial or bulk water.

Plant wall material swells in water to give a coherent structure which changes with the addition of more water to give first a molecular gel and then a particle gel which may, under appropriate conditions, reversibly or irreversibly precipitate, form flocs and even aggregate.

Polysaccharides can form gels with high water contents, depending on their chemistry and species and anatomical source. Pectic substances may form gels but celluloses are more insoluble. With these substances dispersion will be produced by cleavage, either mechanically or by enzymatic hydrolysis of the network. In addition to the dispersion of tightly-packed fibres by natural swelling in water, dispersion will be produced by the presence of small reactive molecules, e.g. acids $(\mathrm{HCl})$ or detergents (bile acids). This process produces a polyelectrolyte system in which colloid particles possess charges balanced by normal-sized small ions, with or without other salts. These ions will influence the degree of swelling.

Water may pass into the capillary structure of the fibre without changing its volume; this process is dependent on the nature of the fibre. Each fibre will have a capacity to hold water which is known as the fibre saturation point and this will be determined by the chemistry of the macromolecule and the peculiarities of the electrolyte concentration and $\mathrm{pH}$ of the surrounding liquid. This capacity to hold water can be demonstrated in the strawberry and cucumber which have a polymer structure which holds more water than an equivalent weight of milk. Carageenan (a seaweed jelly) can form gels which are $99 \%$ water (Rees, 1970 ).

Lignins are much less hygroscopic than the polysaccharides; water is a poor solvent for lignin. The hydrophilic nature of lignin is determined by the oxygencontaining hydroxyl, carbonyl and carboxyl moieties (Kudo \& Kondo, I97I). The viscosity of a lignin solution is low.

Flocculation and aggregation of these gels can occur under varying conditions. 
The widely varying ability of metals to 'salt' out colloids is similar to that of cations with plasma globulins; for example, the ability of cations to precipitate alginates is affected by: (I) the affinity of the metal for alginic acid and (2) the solubility of the metal-alginic acid complex. These complexes are complicated by the presence of sodium (Haug \& Smidtstrød, r965).

Surfactants can modify colloidal systems resulting in dispersion, emulsification or solubilisation of the colloidal systems or in flocculation and coagulation (Alexander \& Napper, 1967). These modifications could be relevant with respect to bile acids which are found in high concentrations in the gastrointestinal tract.

Cation Exchange Capacity. Acidic polysaccharides with uronic acid moieties can act as cation exchangers, i.e. can bind metals. Alginic acid from seaweed is an example; its affinity for cations depends on the source and relative amounts of the acidic sugars, mannuronic acid and guluronic acid. Guluronic acid-rich alginates have a higher affinity for divalent ions than mannuronic acid-rich samples (Haug $\&$ Smidtstrød, 1965). The cation exchange properties are mainly related to free carboxyl groups in pectin and to a lesser extent in hemicelluloses. The cation-exchange capacity is uniform throughout the plant and is characteristic for a species and is relatively independent of external nutritional factors associated with the soil (Knight, Crooke \& Shepherd, 1972).

Organic Adsorption. Vegetable fibre can adsorb bile acids. Conjugated bile acids, e.g. those found in the bile and upper duodenum, are only weakly adsorbed onto the fibre, but unconjugated bile acids, e.g. those found in the colon, are strongly adsorbed. The adsorption is $\mathrm{pH}$ dependent, being greatest at an acid $\mathrm{pH}$, and is possibly influenced by the methoxylation of acidic hydroxyl moieties in the fibre (Eastwood \& Hamilton, I968).

The toxic effects of ionic detergents are counteracted by lucerne but whether this is the result of an adsorption or a dilution effect is not known (Ershoff, 1960). Crude dietary homogenates and certain non-dietary fibres can adsorb pteroylmonoglutamates but not pteroylpolyglutamates. Cellulose is thought to be the binding agent (Luther, Santini, Brewster, Perez-Santiago \& Butterworth, 1965).

Gel Filtration. When a polysaccharide forms a gel a gel-filtration system may develop producing a molecular exclusion capacity. This system has not been studied for vegetable fibre but experiments have been done on cellulose and its susceptibility to enzyme hydrolysis. These suggest that variables such as moisture content of the fibre, the crystallinity of the cellulose, conformation and steric rigidity and the nature of the other molecules with which the celluloses are associated are important (Cowling \& Brown, 1969). The size of the pores of molecular dimensions must dictate the permeability of the surrounding molecules.

Bacteria. If a gel forms, a potential for biological sieving is produced. There is little information about gels formed from vegetable fibre but there is information for cellulose-degrading bacteria and their effect on cellulose fibres. The rate of degradation is dependent on the degree to which the fibrils of cellulose are opened and is complicated by the presence of lignin with the cellulose which can inhibit bacteria passing into the mesh (Berg, Hofsten \& Pettersson, 1972). Many bacteria $32(3) 2$ 
produce extracellular enzymes which may permeate into the gel to act on smaller molecules enmeshed in the matrix.

\section{Biological Implications}

What are the biological implications of these physical effects? First the concentration of electrolytes in the upper gastrointestinal tract is affected by food. Digesta flow through the large intestine is not uniform. The rate of passage of particulate matter is directly proportional to the specific gravity of the material (Hoelzel, 1930). In the rabbit the relative rates of flow of water and particulate matter have a diurnal variation, therefore a soft stool is passed at night and hard faeces during the day (Pickard \& Stevens, 1972). There is, apparently, conservation of water through antiperistaltic flow in the colon in many animals (Elliott \& Barclay-Smith, r904). This water conservation can be lost, in man, in diarrhoea or accentuated in diverticular disease (Findlay, Mitchell, Eastwood, Anderson \& Smith, unpublished results).

Faeces consist of unabsorbed vegetable fibre, bacteria, water, organic and inorganic substances. The unabsorbed vegetable fibre in faeces holds water, some of which is adsorbed to the surface of the fibre (water of hydration); the remainder is trapped in the interstices of the fibre (bulk water). The capacity to hold water is influenced by the chemistry of both the fibre and the liquid phase. In the diarrhoea that can accompany ileal resection, the capacity of the faecal fibre to hold interstitial water is exceeded and a watery stool results. However, the bile acids in the stool remain associated largely with the fibre (Findlay, Eastwood \& Mitchell, I973).

When ingested polysaccharides which have the capacity to adsorb and hold water pass into the colon, there is a bulk laxative effect. Such properties are associated in man with the use of hydrophilic laxatives from agar, methylcellulose, husks from Ispaghul (Piantago ovata L.) seeds of Psyllium (Plantago L.) and gum from Sterculia L. It is certain that the laxative effect of cereal bran is the result of its hygroscopic properties i.e. adsorbing water into the stool. Plant fibre will be hygroscopic and increase stool weight depending on the hydrophilic polysaccharide content. Lignin, which does not bind water, has a costive rather than a laxative effect on the stool. Williams \& Olmstedt (1936) have shown that various plant sources can produce an increase in stool weight. Whole cotton seed produced the least effect on the stool weight, then, with increasing efficiency, cellulose, lucerne-leaf meal, wheat bran, canned beans, maize-germ meal, sugar-beet pulp, cabbage, carrots and finally the most effective agar-agar. There is a twentyfold difference between the least and most effective of these substances.

This increase in stool weight through water-holding capacity will sweep other intestinal contents, normally conserved by reabsorption, out in the stool ; for example, bile acids (Eastwood, Hamilton, Kirkpatrick \& Mitchell, 1973) and electrolytes (Fregly, 197 I) are excreted in amounts which are directly related to the stool weight.

The interactions of mixed polysaccharides are complex and relevant to discussions on the mechanism by which normal faeces are produced. The viscous polysaccharide from Xanthomonas campestris does not form gels but with locust bean (Ceratonia 
siliqua) galactomannan forms a stiff rubbery gel at a concentration at which the latter would not normally gel (Rees, I972).

Cation exchange properties of polysaccharides. Intestinal absorption of strontium is inhibited by orally administered sodium alginate while calcium absorption is not significantly reduced. This inhibition results from the presence of guluronic acid moieties in the alginate (Patrick, I967). Presumably other cations are sequestrated to a variable degree and their loss in the stool is accordingly affected by such binding characteristics. The costive effect of aluminium hydroxide and the laxative effect of magnesium salts could possibly result from distinct abilities to affect gels or by binding to acidic polysaccharide groupings in dietary fibre.

\section{Organic Acid Adsorption}

Bile acids are adsorbed onto fibre in faeces (Gustafsson \& Norman, 1968). This adsorption has been shown to be greater in normal rats than in germ-free animals. The adsorption of bile-acid metabolites to faecal fibre is greater in infected animals on a fibre-containing diet than in similar animals on a semi-synthetic diet. This suggests that the bacterial metabolites of bile acids are more strongly adsorbed than the primary bile acids. Bloomfield $\left(\mathrm{I}_{9} 6_{3}\right)$ has shown that increased fibre content in the diet increases faecal bile-acid output. Cereal fibre is capable of adsorbing bile acids. The effect of feeding cereal fibres on the faecal bile-acid output has not been studied over a sufficient time period to show an effect on faecal bile-acid loss.

In the upper small intestine conjugated bile acids are involved in micellar formation with lipids with no resultant adsorption onto fibre, but in the colon, free bile acids are strongly adsorbed (Eastwood \& Hamilton, 1968). This means that bacterial transformation of bile acids changes the physical state of the bile acids in the intestinal contents by forming derivatives which are strongly adsorbed onto the residue (Norman, 1964).

The effect of fibre has been studied in the rat. The bile salts in the small intestine represent the main physiologically active pool of bile-acid conjugates (Norman \& Sjövall, 1958). Studies of the distribution of bile salts in the rat small intestine indicate that the greatest amount is found in the third quarter which is the area of the small intestine where bile salts are reabsorbed (Boyd \& Eastwood, 1968). The addition of various polysaccharides, sodium carboxymethylcellulose, cellulose and bran to the diet materially increases the amount of intestinal bile salts (Eastwood $\&$ Boyd, 1967); the bile salts are apparently distributed in the intestine in the water phase and a solid phase. If this enhancing effect on bile salts were to be applicable to man it is possible that a diet which contains a significant amount of polysaccharide could result in an increase in the size of the bile-acid pool. This is potentially of great interest in those conditions in which there has been shown to be a relative depletion of bile acids, e.g. in the aetiology of gall stones.

Lucerne and other succulent plants protect mice from the toxic effect of non-ionic, surface-active agents. The highly deleterious effect of ionic detergents Myrge 52, Tween 20 and Tween 60 at dietary levels of $15 \%$ or $20 \%$ were completely counteracted by the coincident feeding of lucerne up to a level of $200 \mathrm{~g} / \mathrm{kg}$ (Ershoff, 1960 ). 
This protective effect was entirely due to the undigested components. Purified cellulose, various grasses, carageenan and sodium alginate were also shown to have some protective activity against these agents. It is not known, however, if these protective effects are due to a dilution or adsorptive effect.

There is no information from man to suggest that dietary polysaccharide gels have a gel-filtration role. However, the presence or absence of dietary fibre in the colon may well be very important in the extracellular enzymic degradation of intestinal contents by affecting the ability of these enzymes to reach small molecules enmeshed in the fibres.

The relationship between dietary fibre and bacteria is complex. It has been shown that bacteria can degrade polysaccharides, e.g. pectin (Werch \& Ivy, I94I), hemicelluloses and celluloses (Milton-Thompson \& Lewis, 1971). It is not known what the effects of these released hydrolysis products have on the intestinal contents or on the bacteria and whether or not these fragments are absorbed from the intestine. Presumably these effects will be controlled by changes in $\mathrm{pH}$ and redox potential resulting from hydrolysis and will depend on the proportion of reducing and acidic sugars present in the parent polysaccharide.

Bacteria hydrolyse water-soluble, conjugated organic acids and the less watersoluble fraction produced, e.g. bilirubin, thyroxine and bile acids will be either precipitated, adsorbed to fibre through hydrophobic bonding or adsorbed by the bacteria; for example, deoxycholic acid $(3 \alpha, \mathrm{I} 2 \alpha-$-dihydroxy-5-cholanoic acid $)$ is adsorbed to vegetable fibre in the stool, while lithocholic acid ( $3 \alpha$-hydroxy-5cholanoic acid) is adsorbed by bacteria (Midtvedt \& Norman, 1972).

There is no information on the effect of dietary fibre content on the spectrum of bacterial flora in the caecum.

Vegetable fibre consists of a heterogenous complex of polysaccharides and lignin. The complex is capable of sequestering water, cations or anions, depending on the chemistry of the constituent macromolecules. Bacteria can degrade both the polysaccharides and the intestinal contents. Vegetable sources will vary in the constituent polysaccharides and lignin, therefore, the effect of these vegetable sources on stool weight and content will vary accordingly.

I am indebted to Drs I. Falkehag, C. T. Greenwood, A. McConnell, W. D. Mitchell and D. A. Rees for discussions and advice.

\section{REFERENCES}

Alexander, A. E. \& Napper, D. H. (1967). Chemy Ind. 1936.

Aspinall, G. O. \& Ferrier, R. J. (I957). F. chem. Soc. 840, 4 I 88.

Berg, B., B. v. Hofsten \& Pettersson, G. (1 972). F. appl. Bact. 35, 2 I 5.

Bloomfield, D. K. (1963). Proc. Natn. Acad. Sci. U.S.A. 50, II7.

Boyd, G. S. \& Eastwood, M. A. (1968). Biochim. biophys. Acta 152, 59.

Cowling, E. B. \& Brown, W. (1969). In Cellulases and their Applications, Adwances in Chemistry Series No. 95 P. I 52 [G. J. Hajny and E. T. Reese, editors]. Washington, DC: American Chemical Society.

Eastwood, M. A. \& Boyd, G. S. (1967). Biochim. biophys, Acta 137, 393.

Eastwood, M. A. \& Hamilton, D. (1968). Biochim. biophys. Acta 152, I65.

Eastwood, M. A., Hamilton, 'T., Kirkpatrick, J. R. Mitchell, W. D. (1973). Proc. Nutr. Soc. 32, 22A 
Elliott, T. R. \& Barclay-Smith, E. (1904). F. Physiol., Lond. 31, 272.

Ershoff, B. H. (1960). F. Nutr. 70, 484 .

Findlay, J., Eastwood, M. A. \& Mitchell, W. D. (1973). Gut 14, 3 r9.

Fregly, M. J. (I97I). Can. F. Physiol. Pharmac. x1, 959.

Gould, S. E. B., Rees, D. A., Richardson, N. G. \& Steele, I. W. (1965). Nature, Lond. $208,876$.

Gustafsson, B. E. \& Norman, A. (1968). Scand. F. Gastroenterology 3, 625 .

Haug, A. \& Smidtstrød, O. (1965). Acta chem. scand. 19. $34 \mathrm{I}$.

Hoelzel, F. (1930). Am. Ұ. Physiol. 92, 466.

Knight, A. H., Crooke, W. M. \& Shepherd, H. (1972). F. Sci. Fd Agric. 23, 263.

Kudo, M. \& Kondo, T. (1971). TAPPI 54, 2046.

Liang, L. H. \& Marchessault, R. H. (1957). F. Polym. Sci. 37, 385 .

Luther, L., Santini, R., Brewster, C., Perez-Santiago, E. \& Butterworth, C. E. (1965). Ala. \%. med. Sci. 2, 389 .

Midtvedt, T. \& Norman, A. (I972). Acta path. microbial. scand. 8oB, 202.

Milton-Thompson, G. J. \& Lewis, B. (1971). Gut 12, 853 .

Notman, A. (1964), Br. F. Nutr. 18, 173 .

Norman, A. \& Sjövall, J. (1958). F. biol. Chem. 233, 872.

Patrick, G. (1967). Nature, Lond. 216, 8 i 5.

Pickard, D. W. \& Stevens, C. E. (1972). Am. F. Physiol. 225, I I6I.

Rees, D. A. (1967). The Shapes of Molecules: Carbohydrate Polymers, Contemporary Science Paperback No. I4. Edinburgh: Oliver and Boyd.

Rees, D. A. (1970). Science \%. 6, 47.

Rees, D. A. (1972). Biochem. F. 126, 257.

Werch, S. C. \& Ivy, A. C. (194I). 7. dig. Dis. 8, IоI.

Williams, R. D. \& Olmstedt, W. H. (1936). F. Nutr. II, 433.

Worth, H. G. J. (I967). Chem. Rev. 67, 465 . 